London, UK; ${ }^{2}$ Clinical Pharmacology, St Thomas Hospital, KCL, London, UK; ${ }^{3}$ Department of Bio-Engineering, University of Amsterdam, AMC, Amsterdam, The Netherlands

Background The mechanisms of the clinically observed phenomenon of reduced angina on second exertion, or warm-up angina, are poorly understood. This study compared changes in central nary blood flow during serial exercise that may contribute.

Methods and Results 16 patients ( 15 male, $61 \pm 4.3$ yrs) with a positive exercise stress test and exertional angina completed the protocol. During cardiac catheterisation via radial access they performed 2 consecutive exertions (Ex1, Ex2) using a supine cycle ergometer. Throughout exertions, distal coronary pressure $\left(\mathrm{P}_{\mathrm{d}}\right)$ and flow velocity $(V)$ were recorded in the culprit vessel using a dual sensor coronary guide wire while aortic pressure was recorded using a second wire. Time to $1 \mathrm{~mm}$ ST depression was longer in Ex2 ( $p=0.003)$ and rate pressure product $(\mathrm{RPP})$ was higher $(\mathrm{p}=0.025)$ confirming warm-up. A $33 \%$ decline in aortic wave reflection $(p<0.0001)$ in Ex2 (see Abstract 41 figure $1 \mathrm{~A}$ ) coincided with a reduction in both tension time index and diastolic time index $(p<0.0001)$. However, the latter was offset by reduced microvascular resistance $\left(\mathrm{P}_{\mathrm{d}} / V\right), \mathrm{p}=0.0002$, and enhanced left ventricular relaxation during Ex2 as suggested by a larger backwardthe intra-coronary signals. See Abstract 41 figure 1B. The energy of the forward compression wave and overall coronary blood flow, as measured by the velocity time integral, did not change.

Conclusions In patients with warm-up angina, exercise induces changes in the aortic pressure waveform, microvascular function and LV relaxation. These combine to reduce afterload without compromising myocardial diastolic blood flow thereby likely enabling improved performance on second exercise.

\section{RETROSPECTIVE CALCULATION OF SYNTAX SCORE IN 200 PATIENTS UNDERGOING ELECTIVE CORONARY ARTERY BYPASS GRAFTING (CABG) AND PERCUTANEOUS CORONARY INTERVENTION (PCI); ARE WE FOLLOWING BEST PRACTICE?}

doi:10.1136/heartjnl-2011-300198.42 haemodynamics, peripheral wave reflection and patterns of corotravelling suction wave $(p=0.01)$ on wave intensity analysis (WIA) of

Discussion The majority of patients undergoing PCI at the FRH have SYNTAX scores in the lowest tertile. There is no difference in the SYNTAX scores in patients having PCI from referral bases within the centre or from outside. In total almost one quarter of all patients undergoing CABG have a SYNTAX score in the lowest tertile. And this rises to almost one third in those patients referred from district general hospitals. Only a small number of these patients have an additional clear indication for CABG over PCI. Furthermore we found that a significant proportion of these do not go through MDT planning. These results may indicate that cardiologists are more likely to bring patients to MDT meetings than surgeons and, according to SYNTAX scoring, more patients are inappropriately having CABG than are inappropriately having PCI. Based on this data in our institution discussing all patients at an MDT and the use of SYNTAX scoring at point of referral would be more likely to increase PCI revascularisation rates.

\section{Abstract 42 Table 1}

\begin{tabular}{|c|c|c|c|c|}
\hline & $\begin{array}{l}\text { Average } \\
\text { SYNTAX } \\
\text { score }\end{array}$ & $\begin{array}{l}\text { \% Patients with } \\
\text { SYNTAX score } \\
\text { in lower third } \\
(0-22)\end{array}$ & $\begin{array}{l}\text { \% Patients with } \\
\text { SYNTAX score } \\
\text { in middle third } \\
(23-32)\end{array}$ & $\begin{array}{l}\% \text { Patients with } \\
\text { SYNTAX score in } \\
\text { higher third }(>33\end{array}$ \\
\hline All Referrals for $\mathrm{PCl}$ & 14.9 & 84 & 11 & 5 \\
\hline $\begin{array}{l}\text { Referrals for } \mathrm{PCI} \\
\text { from within trust }\end{array}$ & 15.0 & 84 & 12 & 4 \\
\hline $\begin{array}{l}\text { Referrals for } \mathrm{PCl} \\
\text { from outside trust }\end{array}$ & 14.8 & 84 & 10 & 6 \\
\hline
\end{tabular}

Abstract 42 Table 2

\begin{tabular}{|c|c|c|c|c|}
\hline & $\begin{array}{l}\text { Average } \\
\text { SYNTAX } \\
\text { score }\end{array}$ & $\begin{array}{l}\% \text { Patients with } \\
\text { SYNTAX score } \\
\text { in lower third } \\
(0-22)\end{array}$ & $\begin{array}{l}\% \text { Patients with } \\
\text { SYNTAX score } \\
\text { in middle third } \\
(23-32)\end{array}$ & $\begin{array}{l}\% \text { Patients with } \\
\text { SYNTAX score in } \\
\text { higher third }(>33)\end{array}$ \\
\hline All Referrals for CABG & 28.8 & 24 & 40 & 35 \\
\hline $\begin{array}{l}\text { Referrals for CABG } \\
\text { from within trust }\end{array}$ & 31.0 & 19 & 34 & 47 \\
\hline $\begin{array}{l}\text { Referrals for CABG } \\
\text { from outside trust }\end{array}$ & 25.0 & 32 & 50 & 18 \\
\hline
\end{tabular}

L J Mullen, R Edwards, R Taylor, B Nyawo. Freeman Hospital, Newcastle upon Tyne

Introduction Cardiologists are generally the gatekeepers of coronary artery disease and have been much criticised for not discussing all patients being considered for revascularisation therapy at an MDT (multi-disciplinary team) meeting or not referring patients with traditional "surgical disease" for CABG. At the Freeman Hospital (FRH), a large cardiothoracic unit in the North of England, patients are typically referred for PCI or CABG by cardiologists working within the Newcastle upon Tyne Trust or from district general hospitals within the network. Patients are not routinely discussed at MDT but can be brought to the weekly meeting at the discretion of the referrer. The recently reported SYNTAX study allows objective quantification of the degree of coronary disease and facilitates an evidence based decision between CABG and PCI. This gives us the opportunity to examine whether elective revascularisation is being performed appropriately at our institution.

Methods We performed a retrospective analysis at the Freeman Hospital. 200 patients who had elective revascularisation between April 2009 and April 2010 were selected. This included 100 cases of CABG and 100 of PCI. Half of each were referrals from other hospitals. Patients' SYNTAX scores were calculated using pre-procedure angiograms. MDT meeting records and patients' notes were reviewed.

Results The average SYNTAX score for patients undergoing elective PCI was 15 , compared to 29 for those undergoing CABG. $84 \%$ of patients undergoing elective PCI had SYNTAX scores less than 22. $35 \%$ of all patients referred for elective CABG had scores greater than 33. The average SYNTAX score for CABG referrals from outside the trust was lower (25) than from within the trust (31).
PROGNOSIS AFTER PRIMARY PERCUTANEOUS CORONARY INTERVENTION FOR STEMI: CAN THE SYNTAX SCORE HELP?

doi:10.1136/heartjnl-2011-300198.43

A J Brown, L M McCormick, N E J West. Papworth Hospital, Cambridge, UK

Background Factors affecting prognosis after primary percutaneous (STEMI) include age at presentation, the presence of diabetes mellitus, left ventricular function and/or cardiogenic shock. Although the debate continues over a strategy of complete revascularisation (immediate or staged) vs culprit-only, little is known about the impact of the extent of coronary disease at presentation on prognosis after PPCI. The SYNTAX score, designed to stratify outcomes in multivessel PCI and CABG, has been validated in unselected populations undergoing elective PCI; to date, no studies have assessed its utility in PPCI. Methods Consecutive patients attending a single UK tertiary centre for PPCI between September 2008 and June 2010 (n=695) were included. SYNTAX scoring was performed by a single trained operator blinded to patient details and outcome. Scoring was validated by analysis of 3 separate cohorts by 2 other experienced operators. Patients were split into 3 subgroups as in the SYNTAX trial (score $\leq 22$ (low, L), 22.5-32 (intermediate, IM) and $\geq 32.5$ (high, $\mathrm{H})$ ), and patient data and outcome measures obtained by interrogation of local and national databases.

Results 671 of 695 patients were included in the analysis with 24 being excluded owing to inability to score (previous CABG, images coronary intervention (PPCI) for ST-elevation myocardial infarction 
unavailable). The ability to allocate a SYNTAX tertile was reproducible between observers $(r=0.94)$. Median scores in the 3 groups were: L 14, IM 26, H 36 (Abstract 43 figure 1A). Although there was no correlation between SYNTAX score and patient sex or diabetic status, there was a linear relationship with patient age $\left(\mathrm{r}^{2}=0.03 ; \mathrm{p}<0.0001\right)$. 1-year absolute survival (Abstract 43 figure 1B) followed SYNTAX score groups: L $94.7 \%$, IM 88.7\%, H 82.1\% ( $p=0.0002)$. Similar results were obtained for freedom from death or unplanned revascularisation $(p<0.0001)$ and death or any revascularisation $(p<0.0001)$.

\section{A}
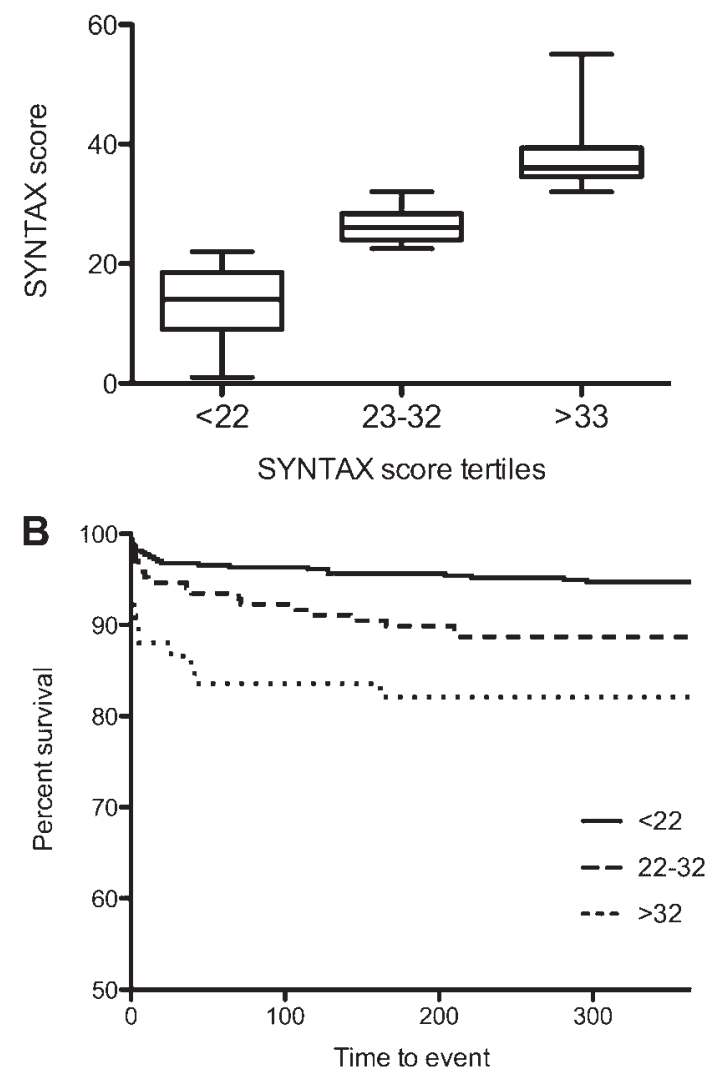

Abstract 43 Figure 1

Conclusions The SYNTAX score, when applied to an unselected population of patients undergoing PPCI for STEMI, provides important prognostic information regarding 1-year survival from death and revascularisation. These findings may provide supporting evidence towards routine complete revascularisation of obstructive coronary artery disease after PPCI.

\section{PRIMARY PERCUTANEOUS INTERVENTION: HAVE WE TAKEN OUR EYE OFF THE MEDICINE BALL?}

doi:10.1136/heartjnl-2011-300198.44

${ }^{1} \mathrm{~J} \mathrm{D}$ Jones, ${ }^{2} \mathrm{E}$ Damm, ${ }^{2} \mathrm{M}$ Nijjar, ${ }^{2} \mathrm{~S}$ Pettit, ${ }^{2} \mathrm{~N}$ M Hawkins, ${ }^{2} \mathrm{R}$ Perry. ${ }^{1}$ University Hospital Aintree NHS Foundation Trust, Liverpool, UK; ${ }^{2}$ Liverpool Heart and Chest Hospital, Liverpool, UK

Introduction Primary percutaneous intervention (PPCI) improves survival in patients with ST elevation myocardial infarction (STEMI). Significant resources have been directed to achieving timely reperfusion throughout the UK. However, intensive medical therapy is of equal importance, with landmark clinical trials demonstrating unequivocal morbidity and mortality benefits from $\beta$-blockers, angiotensin-converting enzyme inhibitors (ACEI), and angiotensin II receptor blockers (ARB). All trials employed rigorous titration to maximum clinically tolerated doses. We examined whether medical therapy is being applied appropriately in patients referred for PPCI. Methods Consecutive patients with STEMI referred for PPCI to a large tertiary centre between 1st March and 1st August 2009 were included $(n=167)$. The case records of all patients were reviewed. Myocardial infarction was diagnosed according to standard criteria. Medications and doses on admission, discharge and follow-up were recorded. Contraindications and limits to dose escalation were noted (symptoms, systolic blood pressure $<90 \mathrm{~mm} \mathrm{Hg}$, heart rate $<50 \mathrm{bpm}$, serum creatinine and potassium).

Results Mean age was $62.0 \pm 11.9$ years, $72 \%$ were male. On discharge, $100 \%$ of patients were prescribed clopidogrel, $95.8 \%$ aspirin, $98.8 \%$ statin, $88.6 \% \beta$-blockers, and $91.0 \%$ ACEI/ARB. However, the inpatient dose of $\beta$-blocker or ACEI/ARB was maximum or clinically limited in only $13 \%$ and $15 \%$ of patients respectively (Abstract 44 figure 1). Outpatient follow-up at a mean of 5.0 months was equally concerning. The majority of patients $(83 \%)$ were neither receiving maximum tolerated doses of $\beta$-blocker or ACEI/ARB, nor received instructions to escalate the dose (Abstract 44 figure 2).

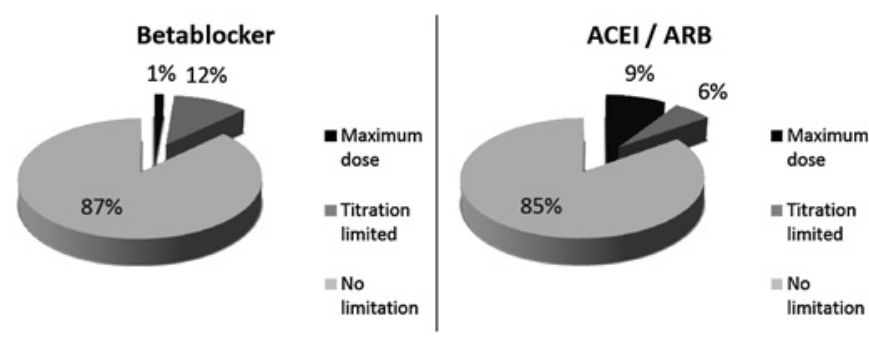

Abstract 44 Figure 1 Inpatient titration of $\beta$-blocker and ACEI/ARB.

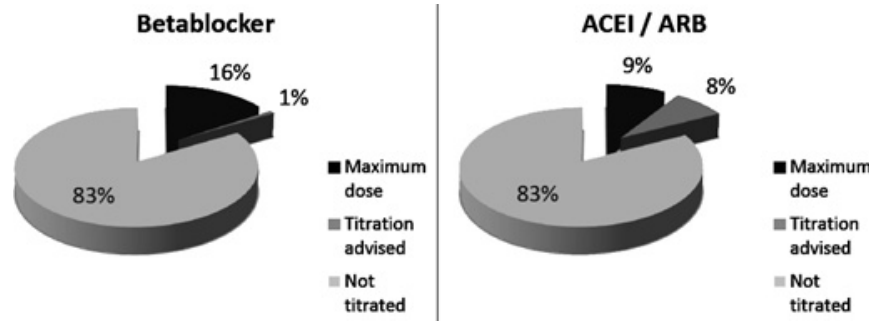

Abstract 44 Figure 2 Outpatient titration of $\beta$-blocker and ACEI/ARB.

Conclusion The national service framework and target driven initiatives such as advancing quality promote "tick box" medicine. Quantitative prescribing of secondary prevention is excellent. Qualitative follow-up and titration is not. Whether suboptimal doses convey the mortality benefits observed in landmark clinical trials is unknown. Frameworks to deliver titration of medical therapy must be explored. Options include nurse or pharmacy led services and expansion of cardiac rehabilitation. Reorientation is needed to focus on both quantity and quality.

\section{SHOULD PRIMARY PERCUTANEOUS CORONARY INTERVENTION BE THE ROUTINE REPERFUSION STRATEGY IN OCTOGENARIANS AND NON-AGENARIANS PRESENTING WITH ST ELEVATION MYOCARDIAL INFARCTION?}

doi:10.1136/heartjnl-2011-300198.45

R Showkathali, E Boston-Griffiths, J Davies, G Clesham, J Sayer, P Kelly, R Aggarwal. The Essex Cardiothoracic Centre, Basildon, UK

Introduction Primary percutaneous coronary intervention (PPCI) has been established as standard therapy for ST elevation myocardial infarction (STEMI). Very few trials have looked at the outcome of PPCI in elderly patients. Even in trials which claimed to have looked 